\title{
New Physics Landmarks: Dark Matter and Neutrino Masses
}

\author{
Farinaldo S. Queiroz $\mathbb{D}^{1},{ }^{1}$ José W. F. Valle $\mathbb{D},{ }^{2}$ Yann Mambrini, $^{3}$ and Giorgio Arcadi ${ }^{4}$ \\ ${ }^{1}$ International Institute of Physics, Universidade Federal do Rio Grande do Norte, Campus Universitario, \\ Lagoa Nova, 59078-970 Natal, RN, Brazil \\ ${ }^{2}$ AHEP Group, Instituto de Física Corpuscular CSIC/Universitat de Valencia Edificio de Institutos de Paterna, \\ C/Catedratico José Beltran 2, 46980 Paterna, Valencia, Spain \\ ${ }^{3}$ Laboratoire de Physique Théorique (UMR8627), CNRS, Univ. Paris-Sud, Université Paris-Saclay, 91405 Orsay, France \\ ${ }^{4}$ Max-Planck-Institut für Kernphysik, Saupfercheckweg 1, 69117 Heidelberg, Germany
}

Correspondence should be addressed to Farinaldo S. Queiroz; farinaldo.queiroz@iip.ufrn.br

Received 22 October 2018; Accepted 25 October 2018; Published 17 December 2018

\begin{abstract}
Copyright (C) 2018 Farinaldo S. Queiroz et al. This is an open access article distributed under the Creative Commons Attribution License, which permits unrestricted use, distribution, and reproduction in any medium, provided the original work is properly cited.
\end{abstract}

The Standard Model has endured several decades and represents the most accurate description of nature we have. Despite the fact that it represents most probably the best description for the electroweak and strong interactions in nature, we have at least two conclusive evidences that the Standard Model is not complete: (i) through the observation of neutrino oscillations we have established nonzero neutrino masses; (ii) a collection of cosmological and galactic observations requires the presence of nonbaryonic dark matter in our universe. The absence of explanations of these two facts within the Standard Model strongly motivates the quest for theory extensions.

Hence, it is paramount to explore new physics directions where these observables and their phenomenological implications are addressed in order to pave the road towards a new Standard Model accounting for all the observed phenomena in nature.

This special issue presents a collection of reviews of some of the most accredited extensions of the Standard Model explaining the origin of neutrino masses and/or the dark matter component of the Universe, as well as original works.

The special feature includes works trying to give an unified description for neutrinos masses in the context of the SU(5) symmetry ("Neutrino Mass, Coupling Unification, Verifiable Proton Decay, Vacuum Stability, and WIMP Dark Matter in SU(5)", by B. Sahoo et al.) as well as those trying to connect neutrino masses to some flavor groups such as
(27) ("Fermion Masses and Mixings in a 3-3-1 Model with $\$ \operatorname{Delta}(27) \$$ Family Symmetry and Inverse Seesaw Mechanism", by A. C. Hernández et al.). In these frameworks, the neutrino mass matrix can get complicated and it is mandatory to investigate its phenomenological impact. The study along this line has been presented by the manuscript "Investigating the Hybrid Textures of Neutrino Mass Matrix for Near Maximal Atmospheric Neutrino Mixing", by M. Singh, which investigated the impact of hybrid textures on the neutrino mass matrix for near maximal atmospheric neutrino mixing. The mixings and oscillations in the neutrino sector were scrutinized in long-baseline experiments by P. Pasquini in "Long-Baseline Oscillation Experiments as a Tool to Probe High Energy Flavor Symmetry Models".

Still, in the context of neutrino mass mechanisms, one popular method to explain neutrino masses is via the addition of Majorana neutrinos which gives rise to neutrinoless double beta decay. The implications have been studied in a model with the $\mathrm{SU}(3) \mathrm{xSU}(3) \mathrm{xU}(1)$ gauge group ("Lower Mass Bound on the $W^{\prime}$ Mass via Neutrinoless Double Beta Decay in a 3-3-1 Model", by A. C. O. Santos and P. Vasconcelos). These works altogether encompass several aspects of neutrino physics going from theory to experimental signatures.

As far as dark matter is concerned, one paper nicely tied neutrino masses to dark matter ("Neutrino Mass and the Higgs Portal Dark Matter in the ESSFSM", by N. Khan), while another focused on having a viable dark matter candidate 
in a popular model where baryon and lepton numbers are promoted to gauge symmetries (" $Z$ ' Portal Dark Matter in the Minimal B - L Model”, by S. Okada). Implications to some recent flavour anomalies are connected to dark matter which is detailed in the work entitled "Anomalies in $b \longrightarrow$ $s$ Transitions and Dark Matter", by A. Vicente. A broader perspective of such dark matter models was detailed in the paper by E. Morgante in "Simplified Dark Matter Models". We also have papers discussing dark matter in the context of wellmotivated supersymmetric models "The Discreet Charm of Higgsino Dark Matter: A Pocket Review”, by K. Kowalska and E. M. Sessolo, and cosmic-ray physics "Impact of Cosmic-Ray Physics on Dark Matter Indirect Searches", by D. Gaggero and M. Valli.

In summary, this special issue contains important works that will allow the reader to be up to date with neutrino and dark matter physics that goes from a more theoretical perspective to an experimental one.

\section{Conflicts of Interest}

The authors declare that they have no conflicts of interest.

Farinaldo S. Queiroz José W. F. Valle Yann Mambrini Giorgio Arcadi 

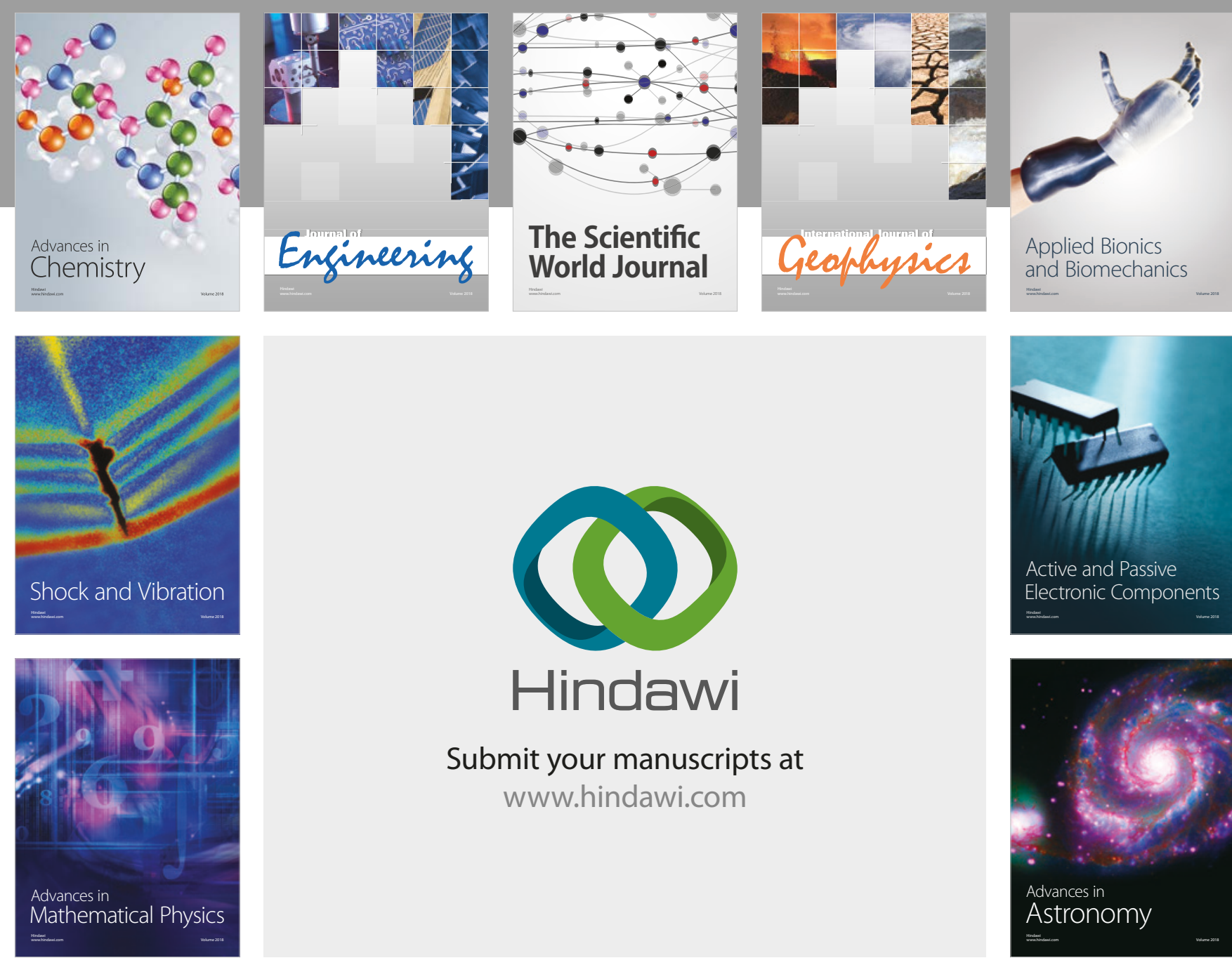

Submit your manuscripts at

www.hindawi.com

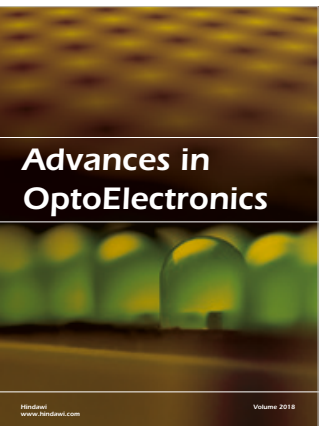

\section{Rotcting Machinery}
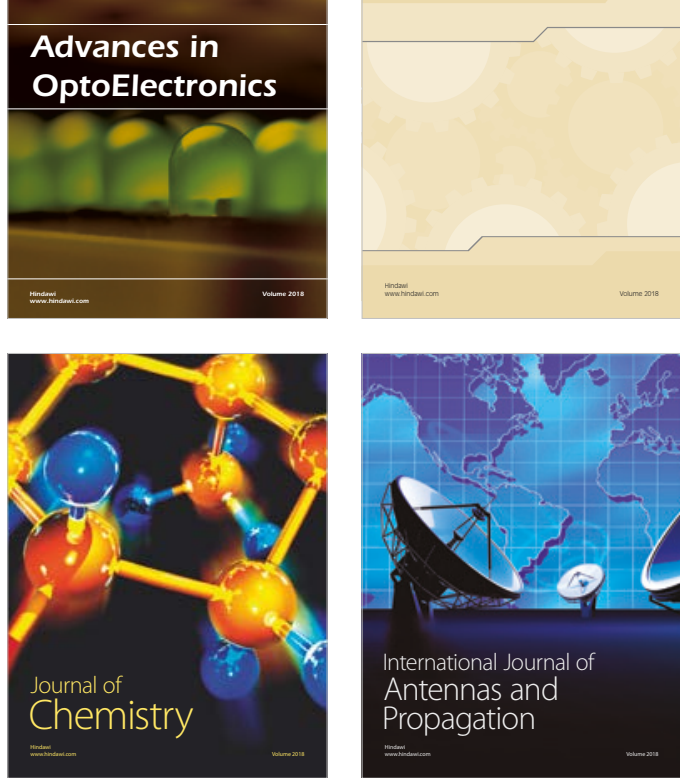

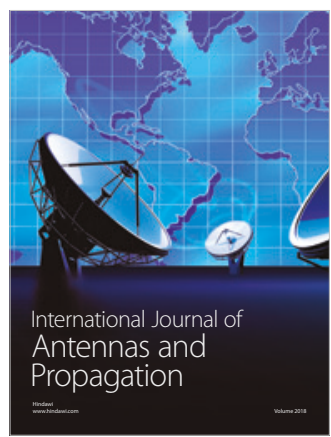

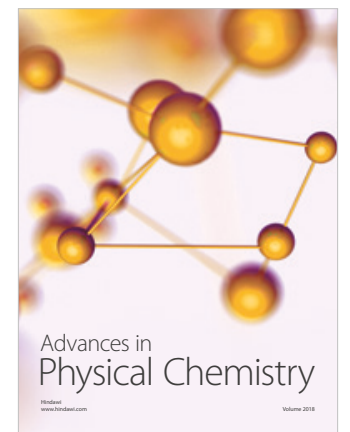

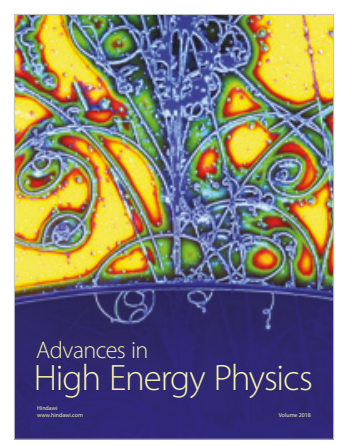

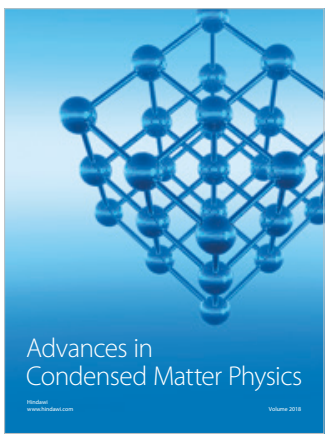

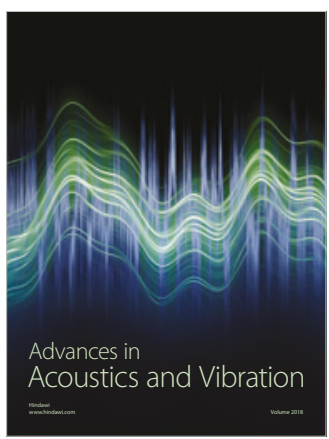

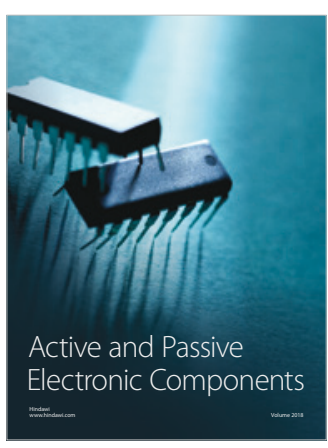
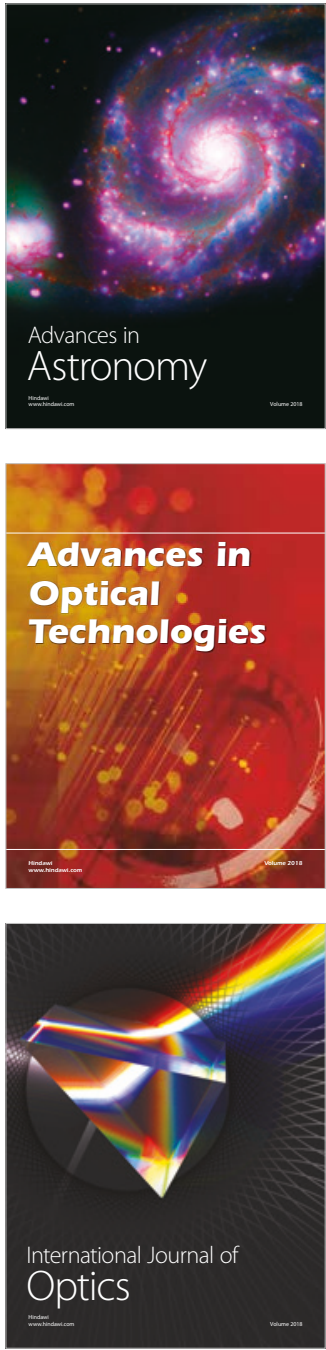